\title{
El gesto político de las filosofías de Heidegger Contribución a un debate actual
}

\author{
FRANCISCO DE LARA \\ Pontificia Universidad Católica de Chile (Chile) \\ fdelara@uc.cl
}

\section{Resumen}

El artículo pretende contribuir a la discusión actual en torno al «caso Heidegger». En concreto, se intentarán mostrar tres gestos políticos correspondientes a tres momentos distintos de la evolución de su pensamiento: el neokantismo, la fenomenología hermenéutica y la ontohistoria. Se planteará hasta qué punto son distintos estos gestos, qué tienen en común y, sobre todo, qué lugar político se le concede a la filosofía en ellos.

Palabras clave: Heidegger, política, fenomenología hermenéutica, historia del ser, elitismo.

\section{The political gesture of Heidegger's philosophies. Contribution to a current debate}

\begin{abstract}
This paper aims to contribute to the current discussion around the "case Heidegger». It shows three political gestures corresponding to three different moments in the evolution of his thought: neo-kantianism, hermeneutic phenomenology and history of Being. We will consider if this gestures are actually different, what do they have in common, and, especially, which political place is granted to philosophy in them.
\end{abstract}

Key words: Heidegger, politics, hermeneutic phenomenology, history of Being, elitism.

Doctor en Filosofía, Albert-Ludwigs-Universität Freiburg y Universidad Autónoma de Madrid. Editor de la Revista Internacional de Fenomenología y Hermenéutica ALEA y director de la colección Filosofía UC (Plaza y Valdés, España). Miembro fundador de la Sociedad Iberoamericana de Estudios Heideggerianos. Autor del libro Phänomenologie der Möglichkeit. Grundzüge der Philosophie Heideggers 1919-1923 (2008), y editor de Entre fenomenologia y hermenéutica. Franco Volpi in memoriam (2011) y de Heidegger. Lógos-Lógica-Lenguaje (2012). Traductor de Problemas fundamentales de la fenomenología 1919/20, de M. Heidegger (2014).

El presente trabajo ha sido realizado en el marco del Proyecto Fondecyt Regular número 1130661 de CONICYT, Chile. 


\section{Introducción}

Cíclicamente resurge una discusión sobre la posición política de Heidegger. Primero, y ya desde 1933, se debatió su nazismo; ahora, probado éste, se busca esclarecer definitivamente su antisemitismo de la mano de una novedad editorial como los Cuadernos negros. ${ }^{1}$. Toda esta controversia, el llamado «caso Heidegger», ha vivido ya diversas etapas, y hay quien divide las posturas al respecto en al menos ocho (Thomä, 2003: 159), lo que da una idea de la complejidad del debate. Lo relevante de esta discusión no consiste, sin embargo, en decidir si la persona Heidegger, o el profesor universitario Heidegger defendieron o compartieron tal o cual ideología. La pregunta de fondo es ante todo si el filósofo Heidegger lo hizo, es decir, si su filosofía misma es o al menos contiene rasgos de lo podría denostarse y repudiarse como contrario a todo pensamiento propiamente dicho. Se pretende decidir, por ende, si su filosofía es derechamente ideología -y además ideología nazi- o al menos contiene suficientes gérmenes de ésta como para que sus fortalezas, sus logros y su influencia filosófica se vean decisivamente opacadas, hasta el punto de resultar ya imposible defender la seriedad de Heidegger como pensador. La cuestión, entonces, atañe al gesto político que su filosofía exhibe o conlleva, a los elementos políticos que su pensamiento pone en marcha implícita o explícitamente. Definir estos gestos con justicia, sin intenciones de antemano apologéticas o condenatorias, y hacerlo sobre la base de un conocimiento adecuado de su pensamiento resulta de la mayor importancia para un debate que, a mi entender, va más allá de Heidegger y permite reflexionar sobre el lugar político de la filosofía.

Las siguientes páginas pretenden contribuir sobre todo a esta última discusión, si bien partiendo también aquí del caso Heidegger. En concreto, intentaré mostrar tres gestos políticos correspondientes a tres momentos distintos de la evolución de su pensamiento: el neokantismo, la fenomenología hermenéutica y la ontohistoria². Mi intención es plantear

1 En concreto, sus escritos titulados Überlegungen, correspondientes a los volúmenes 94, 95 y 96 de la edición integral (Gesamtausgabe) de sus escritos y recientemente publicados por Peter Trawny. El propio Trawny (2014) ha debatido dicho antisemitismo en su libro Heidegger und der Mythos der jüdischen Weltverschwörung, editado ya por segunda vez en el transcurso de unos pocos meses.

2 Los gestos corresponden a manifestaciones del pensamiento de Heidegger en los años 1915, 1919 y 1933, por lo que este análisis no pretende ser exhaustivo y es susceptible de ser completado e incluso matizado considerando otros momentos. Me parece, no obstante, que los gestos aquí seleccionados son absolutamente característicos y permiten: a) mostrar el lugar político que, según Heidegger, ocupa la filosofía, y b) explicitar los presupuestos políticos de esta manera de entender la filosofía. 
hasta qué punto son estos gestos distintos, qué tienen en común y, sobre todo, qué lugar politico se le concede a la filosofía en ellos. Empezaré identificando esos gestos para seguir después con un análisis crítico de los mismos.

\section{Identificación de los gestos políticos}

\subsection{La conferencia de habilitación de 1915 y las primeras publicaciones de Heidegger}

La primera manifestación oral del trabajo filosófico de Heidegger fue su conferencia de habilitación sobre El concepto de tiempo en la ciencia bistórica, dictada el 27 de julio de 1915 en la universidad de Friburgo y publicada un año más tarde (Heidegger, 1978a: 413-433). La conferencia se inicia con unas palabras sobre el nuevo impulso metafísico que la filosofía científica estaría adquiriendo en esos años a fin de no verse reducida a una estrecha especialización que renuncia a las preguntas motivadoras y orientadoras del filosofar. Heidegger interpreta este impulso metafísico como una expresión de la «voluntad de poder de la filosofía» (Heidegger, 1978a: 415), esto es, de su negativa a limitarse a problemas epistemológicos y sus más altas aspiraciones a dirigir la cultura. Estas palabras se insertan de pleno en el esfuerzo de una parte de la filosofía alemana del momento por no permitir que fueran otros impulsos, derivados del predominio de las ciencias naturales, quienes guiaran y transformaran al hombre. Dicha pretensión de limitar la influencia del positivismo y el modelo de las ciencias naturales en el estilo de vida y las convicciones de la cultura occidental se realiza en estos años de forma científica, esto es, mediante un trabajo de delimitación de las pretensiones absolutas de dichas ciencias y una mostración del sentido propio de las ciencias humanas, en la línea de lo iniciado por Lotze y Dilthey y continuado a su manera por el neokantismo y Husserl. En concreto, Heidegger pretende aquí contribuir en esta dirección mostrando los límites de la concepción física del tiempo y su disparidad de principio con la concepción del tiempo propia de la historia. Esta forma científica, rigurosa, de proceder es la que daría carta de legitimidad a las pretensiones de poder de la filosofía, evitando cualquier arbitrariedad. De ahí que sea preciso seguir trabajando en problemas epistemológicos concretos, pero sin perder de vista la necesidad de una «fundamentación metafísica última» (Heidegger, 1978a: 415). Para lo que aquí nos importa, basta con subrayar tanto el impulso decididamente político que Heidegger reconoce a su trabajo filosófico como la forma que tomaría de facto este impulso en cuanto encarnación de un verdadero espíritu crítico y científico. 
Este espíritu de lucha y combate por unos ideales no le era ajeno a Heidegger, formado en un ambiente combativamente católico que veía con malos ojos varias de las transformaciones que acompañaron al predominio de las ciencias naturales, sus aplicaciones técnicas y la consiguiente transformación del entorno natural y social. De hecho, su primer escrito sobre filosofía -Das Realitätsproblem in der modernen Philosophie (1912) - aparece en el anuario de la Sociedad Görres (Heidegger, 1978a: $1-15)^{3}$ y todas sus publicaciones siguientes anteriores la tesis doctoral -en su mayoría reseñas- son publicadas por la revista Literarische Rundschau für das katholische Deutschland ${ }^{4}$. En estos primeros años marcados por la Kul-

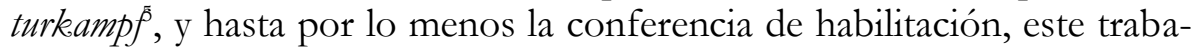
jo político adopta una forma científica. Se considera necesario realizar un estudio serio y riguroso de los problemas filosóficos; estudio que, sin embargo, no constituye un fin en sí mismo, sino que apunta en el fondo a la necesidad de promover unos ideales y fines como intrínsecamente humanos, conservándolos y defendiéndolos ante la amenaza de una sociedad de hombres desligados entre sí y ajenos a estos ideales, movidos ya únicamente por intereses materiales y desprovistos de todo lazo comunitario y valórico.

\subsection{Los primeros cursos friburgueses. "Ciencia y reforma de la universidad"}

Algo más tarde, en los primeros cursos que imparte en la misma universidad de Friburgo, Heidegger parece haber tomado distancia de esta labor combativa, y asignarle a la filosofía un rol mucho menos directo en la transformación política de la sociedad. Esto se aprecia de forma clara en su primer curso, que se inicia justamente con una toma de postura ante acontecimientos derivados del final de la primera Gran Guerra. En efecto, varias fueron las voces que en ese momento clamaron por una renovación de la universidad y del modo como se cultivaban y se

3 Sobre las relaciones de Heidegger con esta importante sociedad en el contexto de la Kulturkampf, véase Ott (2004), reeditado después en Ott (2005). En un trabajo bastante anterior, Ott (1986) había estudiado las becas que Heidegger recibió en sus años posdoctorales, una de las cuales provino de la iglesia friburguesa (la beca von Schaetzler) y otra de la misma sociedad Görres. En castellano puede consultarse Ott (1993).

4 La primera publicación de Heidegger, sobre la inauguración de un monumento a Abraham de Santa Clara, data de 1909 y ve la luz en el Heuberger Volksblatt, diario antimodernista fundado en Messkirch en 1899 como oposición al liberal Oberbadischer Grenzbote. (Heidegger, 1978b: 1-3).

5 Sobre Heidegger y el movimiento antiliberal, antimodernista y ultramontano surgido en el sur de Alemania a raíz del Concilio Vaticano I, puede verse ahora el primer capítulo del libro de Wolfe (2013). 
enseñaban la ciencia y la filosofía, considerando que dicho modo debía estar acorde al rol que tales disciplinas pueden y deben cumplir en la transformación de sociedad. En este sentido, baste con recordar a modo de contraste la implicación de Walter Benjamin, estudiante en Freiburg durante los mismos años que Heidegger, en los movimientos de juventud y estudiantiles ${ }^{6}$.

Heidegger se posiciona al respecto de estas preocupaciones y debates ya en las primeras palabras de su primer curso justo después de la guerra, las Consideraciones previas sobre Ciencia y reforma universitaria (Heidegger, 2005: 3-6). En dicho curso, dirigido a estudiantes que habían participado en la contienda, Heidegger se propone determinar el carácter y la tarea de la filosofía, y lo hace justamente a partir de una delimitación de sus pretensiones de ofrecer una visión del mundo. Ya no se trata, como en los primeros años, de combatir y limitar los efectos de la cosmovisión científica de orientación naturalista mediante el fomento de una visión católica o científico-espiritual del mundo. En el extremo opuesto a esta posibilidad, Heidegger pasa ahora a denunciar toda cosmovisión, también la científica, como fundamentalmente ajena a la filosofía ${ }^{7}$.

Por este camino la filosofía parecería haber perdido la motivación y finalidad política que Heidegger le asignaba en los años anteriores, renunciando a ejercer alguna influencia sobre el mundo actual. En efecto, la respuesta de Heidegger al problema de una posible crisis de la ciencia, la filosofía y las instituciones donde éstas se desarrollan y enseñan no contempla ninguna propuesta de organización o cambio concreto en las dimensiones públicas y compartidas de éstas, ni en el ideal de ser humano que debería moverlas ni en las finalidades que deberían tener. Lo que Heidegger propone es, antes bien, un camino de seriedad personal que permita a cada cual, individualmente, convertirse en el tipo de existencia que estas disciplinas ejemplifican. Al igual que en los años anteriores, también aquí la ciencia y la filosofía son entendidas por Heidegger

\footnotetext{
6 Sobre Benjamin y los movimientos de juventud, puede consultarse el reciente libro de Eiland \& Jennings (2013), especialmente su segundo capítulo. No está de más mencionar en este contexto la carta a Scholem de 1916 en la que Benjamin critica la forma como Heidegger trata, justamente en el artículo que recoge su conferencia de habilitación, el tiempo histórico y su diferencia del tiempo mecánico. Véase Benjamin, 1995: 344 y, sobre ello, Regehly (2002) y Vargas (2012: 87).

En ese sentido, afirma que «la tentación cultivada por doquier en las ciencias particulares [...] de armarse de una "concepción del mundo" científica a través de la gramática fraseológica de una filosofía contaminada ejerce una fuerza paralizante, al igual que el "espíritu activo" de los jóvenes que se dedican a escribir» (Heidegger, 2005: $5)$.
} 
como tipos ideales, pero no inciden ya en el carácter que puedan adoptar las distintas culturas y sociedades, sino únicamente en formas individuales de vida. La investigación deja de concebirse como encarnación de valores que pueden modelar la fisionomía de una cultura y se convierte en una incumbencia radicalmente personal que demanda seriedad y compromiso en primera persona del singular.

El desplazamiento del lugar de la filosofía científica es tal que ya no se la entiende como una necesaria toma de distancia crítica con las dimensiones comunes de la vida, sino como una verdadera ruptura con todas esas dimensiones en cuanto ya niveladas y poco auténticas. La misma idea de cultura que había guiado los planteamientos neokantianos que Heidegger aceptaba en su conferencia de habilitación es rechazada de forma cada vez más marcada en estos años ${ }^{8}$. La filosofía no detenta ya ningún afán de transformación o salvación de la cultura en cuanto dimensión común y pública; lo que la filosofía transforma es la existencia personal, y lo hace justamente dando la espalda a toda esfera común. La única comunidad posible desde estos postulados sería justamente la comunidad de los filósofos, es decir, la comunidad de los que se esfuerzan por vivir propiamente, con plena responsabilidad existencial, haciéndose cargo de la problematicidad de la existencia y evitando toda tendencia al aligeramiento y la huida de sí. Según considera este Heidegger, en la filosofía, como en el cristianismo primitivo, cada cual se convierte en un problema para sí mismo ${ }^{9}$ y dedica su esfuerzo intelectual-vital a asumir esa problematicidad y atreverse a vivir en ella, «despierto» (Heidegger, 1999: 7.15).

Sin embargo, y al contrario de lo que podría parecer, toda esta nueva concepción del sentido, la motivación y el rol fundamental de la filosofía no implica una renuncia a la idea de que ésta puede en efecto producir cambios y renovaciones en el mundo, en la esfera común y sus instituciones. Lo que cambia es tan sólo la manera como lo hace, por lo que se postula aquí una nueva estrategia de acción política de la filosofía que

8 Una clara muestra de este cambio de perspectiva se encuentra en sus "Anotaciones a la Psicología de las concepciones del mundo de K. Jaspers", donde se afirma la necesidad de resolver a fondo «el problema de lo que nosotros mismos 'somos'» y se pregunta si los filósofos de su tiempo no estarán «embargados por una preocupación por salvar la cultura que nadie [les] ha encargado» (Heidegger, 1996: 5).

9 Esta apropiación de Heidegger del célebre «quaestio mihi factus sum» agustiniano se encuentra literalmente (Heidegger, 1995: 178. 209). Sobre la interpretación y el diálogo con el cristianismo del joven Heidegger existe muchísima literatura. Como introducción a la temática, con el acento puesto en los aspectos del cristianismo de San Pablo y San Agustín que Heidegger adapta a su proyecto filosófico, me permito remitir a De Lara (2007). 
será la característica de Heidegger en lo sucesivo. Según ella, los cambios políticos tienen lugar de forma indirecta y lenta, por un camino adecuado sólo para unos pocos, mucho más largo y silencioso que el de la discusión pública. En otras palabras, la convicción ahora defendida afirma que no son los programas de renovación de la cultura lo que lleva a auténticas transformaciones, sino un estilo radical y verdadero de existencia. Como dice Heidegger en esas primeras palabras del mencionado curso de 1919, «sólo la vida hace 'época', y no el bullicio de frenéticos programas culturales» (Heidegger, 2005: 5). Heidegger llama por ello a una total honestidad (W abrhaftigkeit), al trabajo vocacional silencioso: a volverse esencial, tal y como demandaba A. Silesius (Heidegger, 2005: 5). Incluso cita el Evangelio (Mt 19,12) para dejar claro que esto sólo lo podrá entender quien siga el mismo camino, que la única comunidad aquí vislumbrada es la de quienes comparten el mismo estilo serio, esencial y silencioso de vida en la verdadera investigación, esto es, en la investigación radical que hace problema de uno mismo. El filósofo maestro se convierte ante todo en ejemplo de existencia, encarnación del ideal de vida que nos mueve en forma de vocación. En estas primeras palabras del curso, toda una declaración de intenciones, Heidegger pone al investigador en el mismo plano que el religioso, que guarda un respetuoso silencio ante el misterio, o el artista que, siguiendo la máxima de Goethe, se dedica a configurar y evita el parloteo (Heidegger, 2005: 5).

Este trabajo silencioso y poco visible, inaparente, es ya aquí considerado el modelo de lo que finalmente lleva a las verdaderas transformaciones. Con ello, se invita a los estudiantes a una reconcentración y seriedad que los aparte de los movimientos políticos concretos, de reivindicaciones e intentos de cambio específicos ${ }^{10}$, se los conmina al silencio hasta volverse esenciales para, entonces sí, generar un cambio incluso a su pesar, por la pura fuerza de su ejemplo vivo ${ }^{11}$. Es el espíritu el que revoluciona de la mano de sus grandes ejemplos, nunca la mayoría o los directamente afectados por un estado de cosas concreto. Lo que debe ser trasformado, por su parte, no son nunca aspectos concretos, materiales; no se trata de pequeñas reformas, sino tan sólo de grandes transformaciones a partir del espíritu encarnado por hombres grandes ellos mismos.

\footnotetext{
10 Heidegger (2005: 4) toma clara postura al respecto al afirmar que «la reforma universitaria de la que tanto se habla va totalmente errada y desconoce por completo toda auténtica revolución del espíritu, cuado ahora se enzarza en proclamas, asambleas de protestas, programas, órdenes y alianzas: medios contrarios al espíritu y al servicio de fines efímeros».

11 Sobre la función del ejemplo en esta transformación de la vida, Heidegger (2005: 5.
} 
Como puede apreciarse, en estos años la relación con el ámbito político adopta en Heidegger la forma de un desprecio de lo público en general: como dimensión interpretativa de la vida y también como ámbito de comunidad y de posibles transformaciones de las estructuras que nos afectan en lo común. Se llama más bien a un rechazo de todo ello y a una individualización radical que nos permita entonces, desde ahí, establecer una «comunidad de lucha» ${ }^{12}$ de unos pocos dentro de esta comunidad pública. Esta lucha se proyecta como la única posibilidad para conducir a una transformación que no sea no parcial sino total, no externa sino interna, no material sino espiritual. En definitiva, Heidegger parece estar pensando a partir del modelo de la transformación interior que finalmente tiene efectos sobre el mundo, en la forma de conversión de otros a este mismo estilo de vida por la fuerza del ejemplo carismático.

\section{3. "Caminos para el debate". El rol político de la filosofía en la ontohistoria}

Según lo visto hasta el momento, en sus primeros textos Heidegger lucha por un estilo de vida espiritual-católico que se siente amenazado por el «liberalismo» y por la dispersión y frivolidad de la vida urbana; en la conferencia de habilitación se afirma la voluntad de poder de la filosofía para «dominar» (meistern) (Heidegger, 1978a: 415) la cultura ante esa misma amenaza de naturalización y mecanización de la vida. Por su parte, en los primeros cursos de Friburgo esta amenaza contra el espíritu no es combatida ya en la arena pública, la lucha es ahora interna y toma la forma indirecta de una dedicación plena y radicalmente honesta a la propia tarea. El filósofo no es ya un agente político directo, sino un héroe del espíritu que responde a la amenaza con aristocrático desprecio y conciencia de que su tiempo llegará. Las transformaciones se vuelven de este modo mucho más lentas y silenciosas, a menudo se dice que serán precisas generaciones enteras de trabajo serio y ejemplarizante hasta que se logre un verdadero cambio ${ }^{13}$. Así pues, y a pesar del supuesto desprecio por lo público, el auténtico agente político es para Heidegger el filósofo y su acción es indirecta, a largo plazo. Por alguna razón se confía y se afirma que en ello se cifra la agencia real de la historia, lo que hace época y no ruido.

\footnotetext{
12 Hago referencia aquí, claramente, al programa de «auténtica» reforma universitaria que Heidegger y Jaspers compartieron en estos años bajo el nombre de una «comunidad de lucha» entre ambos (Heidegger \& Jaspers, 1992).

13 Véase, al respecto, Heidegger (2005: 4; 1993: 6. 22-23. 177).
} 
Este mismo motivo de la lentitud e invisibilidad pública de lo que mueve la historia de modo silencioso e inaparente, apreciable tan sólo por los pocos -en este caso filósofos y artistas-, se mantiene en el pensamiento de Heidegger posterior a la Kehre. También el motivo asociado a éste de la amenaza, que ahora se presenta ya más globalmente como la amenaza de Occidente, esto es, de lo que se postula como el modo de existencia occidental. En el fondo se trata del mismo motivo de una sociedad guiada por el espíritu y amenazada por un materialismo tecnócrata representado en este caso por otras potencias mundiales. En su texto de 1937 titulado Caminos para el diálogo (Wege zur Ausprache) presenta a Francia y Alemania como «los dos pueblos vecinos que más esencialmente han participado en la configuración histórica y espiritual de Occidente» (Heidegger, 1978b: 15) -dejando de lado la herencia griega, latina, árabe, italiana, española, inglesa...- y entre los que sin embargo tan difícil parece ponerse de acuerdo en una hora del mundo en la que Occidente se ve amenazado y precisa salvación (Heidegger, 1978b: 16). El caso es que, una vez más, ese ponerse de acuerdo no tiene para Heidegger la forma de un llegar a acuerdos sobre aspectos concretos del tipo que sea, sino reconocer y confrontarse con lo que se postula como el carácter esencial del otro pueblo. Heidegger incluso sanciona ese llegar a acuerdos concretos como un entendimiento impropio (Heidegger, 1978b: 16. 21); de lo que se trataría es de recordar al otro pueblo su particular destino a fin de combatir la amenaza común de desconcierto y desarriago (Heidegger, 1978b: 16. 20).

De nuevo apreciamos que la posible comunidad tiene para Heidegger el carácter de una comunidad de lucha contra una amenaza, comunidad surgida de un comprender la propia tarea a la que el sujeto político se ha dado. Aquí los sujetos políticos no son ya las culturas ni los individuos heroicos dedicados a la problematización de la existencia, sino los pueblos movidos por un destino, esto es, por una tarea. Ese destino depende, en el fondo, de la 'ontohistoria', de la historia del ser. Los pocos que entienden esa historia y la configuran para sus pueblos son los artistas y los filósofos (Heidegger, 1978b: 17). De este modo, en la filosofía se estarían abriendo formas de ver, disposiciones y vías que prefiguran siempre el modo fundamental de vivir de los pueblos y de Occidente todo $^{14}$. Por ese motivo, la filosofía sería el lugar donde, una vez más, se

14 En ese sentido, afirma Heidegger que «el saber auténticamente filosófico... [es] el saber anticipador que abre nuevos ámbitos y respectos de preguntas acerca de la esencia de las cosas, esencia que se oculta siempre de nuevo. Justamente por eso, dicho saber no puede volverse nunca utilizable de forma inmediata. Éste tiene efecto sólo mediatamente, pues la meditación filosófica prepara nuevas vías de la mirada y nuevas varas de medir para todo comportamiento y decisión. Así, de antemano y oculta para toda per- 
jugarían las transformaciones más profundas y basales, si bien de forma mediata, lenta e inaparente.

\section{Análisis crítico de los tres momentos}

Como puede apreciarse en este repaso necesariamente condensado e incompleto, las consideraciones políticas que propone Heidegger en los dos últimos momentos analizados -los más claramente representativos de su pensamiento- se plantean siempre como radicales y originarias, esto es, no reducibles al ámbito de la discusión política concreta, sino mostradoras de una esfera previa de la que, sin embargo, dependería incluso lo concreto y cotidiano (en cuanto pálido reflejo ya enajenado de lo que de verdad determina e impera). Así pues, lo que le da a las cosas su verdadera y última fisonomía no sería para este pensador algo perteneciente al plano social y político, sino algo previo: radicalmente individual en el primer Heidegger; impersonal y no humanamente determinable en el segundo. En el Heidegger posterior a la Kehre el papel de esa instancia configuradora lo juega el Ser en su acontecimiento, al que los humanos no responden individualmente sino en cuanto pueblos. Lo más decisivo de estos pueblos, sin embargo, serían sus fuerzas creadoras, desplegadas en el ámbito del arte y la filosofía. En esto coinciden las consideraciones del primer Heidegger con las de este segundo: lo histórico debe entenderse como obra de grandes hombres dedicados a tareas de carácter espiritual-creativo. Lo social es, en sus rasgos esenciales, el resultado o el reflejo de la acción de tales hombres. Así pues, la dimensión material y política no es considerada como el lugar fundamental del que surge y al que vuelve de algún modo la filosofía, sino como algo secundario y además resultado en lo esencial de fuerzas pre-políticas, entre las que se encuentra en un lugar señalado la misma filosofía.

Esta acentuación de la dimensión pre-política como ámbito originario lleva a un desprecio de los acontecimientos y a una lectura espiritualizada e idealizada de la historia. Las posturas políticas que es posible adoptar desde aquí estarán caracterizadas por un rechazo de todo cambio y propuesta en el plano verdaderamente político -por considerarlos inesenciales- y por una tendencia al extremismo o al fatalismo. El extremismo es más propio del primer Heidegger, dado que todavía se considera en manos de unos pocos la conversión general de la sociedad a unos

secución de la utilidad, la filosofía señorea la postura y la manera de proceder del existir histórico del hombre. La filosofía es el saber inmediatamente inútil, pero no obstante señorial, de la esencia de las cosas» (Heidegger, 1978b: 18). 
ideales y una tarea común. El fatalismo es más propio del segundo, pues en última instancia todo depende del esenciarse de la verdad del ser, de modo que 'sólo un dios puede salvarnos' de esas amenazas que constituyen siempre la contraparte de la lectura heideggeriana del ámbito público.

Vale la pena detenerse en este último punto, pues resulta del todo característico. Desde el principio hemos visto que el trabajo filosófico se realiza para Heidegger siempre a la sombra y como respuesta a un estado del mundo considerado amenazante. En general es la modernización y la tecnificación, la pérdida de lazos valóricos o esenciales, lo que ocupa este lugar negativo. El panorama, entonces, es siempre el de un enemigo externo que se expande por doquier y que sólo unos pocos hombres espirituales y profundos pueden combatir con su trabajo, guiado hacia lo esencial y por-venir. Como puede apreciarse, nos las tenemos aquí con un elitismo político sólo parcialmente disimulado de indiferencia política. Dicho elitismo conlleva una justificación de la lejanía y efectiva indiferencia para con los asuntos y problemas reales: injusticias, abusos, explotación, pobreza, etc. ${ }^{15}$. Nada de eso sería asunto del filósofo, dirigido a lo invisiblemente determinante, no a lo coyuntural y derivado.

Lo que su trabajo pretende alcanzar es un arraigo en medio del desarraigo que se expande, una comunidad de los que luchan por lo mismo con su trabajo y su existencia toda, los que comparten la misma visión de esa forma de vida a salvaguardar y de la amenaza que los conmina a la acción. De este modo, todos aquellos que no forman parte de esa supuesta comunidad no son vistos únicamente como ingenuos que no comprenden la hora del mundo, sino también como fracción cómplice de lo que expande la amenaza. En lugar de reconocer la complejidad social y la diversidad de posturas e intereses que tienen lugar en la vida pública, tenemos aquí una visión simple que divide a la sociedad entera en dos bloques abstractos: un escaso grupo de auténticos iluminados y una multitud ciega que los rodea y amenaza. Cabe preguntarse qué sucedería si una visión así lograra en efecto tener poder político y estar en condiciones de establecer disposiciones y medidas. Está claro que Heidegger diría que esto es imposible, pues el imperar de la filosofía es

15 Basta repasar brevemente la forma como Heidegger se refiere a estos asuntos -la pobreza y la escasez de vivienda tras la II Guerra Mundial, los campos de concentración, etc.- para hacerse una cabal idea de un gesto que sólo cabe entender como ceguera insensata o como ideología encubridora. En efecto, a pesar de su pretensión de distancia, Heidegger no cesa de referir a fenómenos coyunturales, y de hacerlo siempre con la misma estrategia en dos pasos: un primer paso que decreta lo superficial del modo habitual de tratarlos y un segundo paso que desrealiza tales fenómenos mediante su incorporación a los esquemas fijos de interpretación que dominan en su filosofía de ese momento. 
inaparente y no inmediato, pero la cuestión es la imagen de la sociedad que desde aquí se proyecta y las consecuencias políticas que esta visión podría tener en caso de que alguien con verdadero poder se la tomara en serio.

Por fortuna, casi nadie se toma en serio a los filósofos y lo que mueve el mundo no parecen ser la poesía o la filosofía, precisamente. Y quizá de aquí surja un inevitable conflicto que las obliga a tener una posición incómoda en el mundo y a guardar con éste relaciones tensas y difíciles. Ellas no siguen la lógica del mundo dominante -son por esencia críticas y disfuncionales- pero tampoco son ajenas a ese mundo, sino que siempre hablan de él y también a él. La filosofía parte del ámbito público y vuelve a dicho ámbito; incluso las filosofías pretendidamente apolíticas son publicadas y pretenden tener un efecto en sus lectores. Así pues, las pretensiones de estar por encima o al margen de todo esto no pueden sino resultar ambiguas. A pesar del gesto profundo y trascendente, filosofías como la de Heidegger tienen -y no pueden sino tener- intención de influir y de ser consideradas por otros, ponen en marcha ideales de vida individual y común, dan líneas directrices, pretenden ser orientadoras, ofrecen una lectura del mundo, juzgan, critican y ensalzan, apoyan y rechazan. La cuestión, entonces, no es si la filosofía es política o no, sino el modo como lo es.

Si consideramos de qué manera es política la filosofía de Heidegger, es preciso concluir que, como no puede ser de otra manera, su trabajo filosófico se inserta estructuralmente en un mundo y a la vez supone una cierta ruptura crítica con él. No obstante, se realiza de tal manera que finalmente se presenta como si no tratara de este mundo ni pretendiera transformación práctica alguna del mismo. Su discurso denota gran sagacidad, brillantez e incluso genio, así como otras muchas virtudes propias del trabajo intelectual. En general, no obstante, acaba tendiendo a la construcción, intenta encontrar algo supramundano que dé cuenta última de lo mundano sin serlo ello mismo (por lo que manifiesta una tendencia metafísica y dualista) y pretende además que esa instancia tenga algún poder normativo sobre el mundo en general; sin que por lo demás se preocupe por el mundo concreto, si no es a través de sus propios esquemas de pensamiento, que lo alejan precisamente de dicho mundo. Como se ve, Heidegger sería un caso sin duda particular y complejo, pero un caso al fin y al cabo, de una forma de hacer filosofía con tendencia al escapismo y la indiferencia política -pero que a menudo, y desde luego en el caso de Heidegger, sustenta posiciones políticas elitistas y conservadoras. 


\section{Conclusión}

En un texto por el 80 cumpleaños de Heidegger, Hannah Arendt narraba la fama de éste ya en sus primeros años y se refería a él como a un «rey oculto» (Arendt, 1969). A la vista de lo analizado, podemos concluir que el nombre le conviene a la perfección. Heidegger se ve a sí mismo como otro filósofo rey, pero ahora ya oculto y aislado, sin poder efectivo, dueño tan sólo de un imperio invisible, inaparente. Un rey que considera indigno mezclarse en los asuntos del pueblo, pero que no deja de hablar de ellos y de hacerlo siempre con el mismo gesto. Apártense del ruido, nos dice, conviértanse al partido de los pocos; dedíquense a lo esencial, a la filosofía, porque es en ella y no en el mundo práctico donde tienen lugar las transformaciones. Este gesto, que espero haber delineado suficientemente a partir de los tres momentos analizados, define la función política de la filosofía, su lugar y su posible influencia en el mundo público. Al considerar cómo entiende Heidegger esta función se vuelve manifiesto, ante todo, cuál es la posición política de la filosofía del propio Heidegger. Mostrar esto en el contexto del actual y tan embrollado debate en torno al caso Heidegger tal vez pueda servir a modo de contribución.

\section{REFERENCIAS}

-Arendt, H. (1969). Martin Heidegger ist achtzig Jahre alt. Merkur, 23 (10), 893902.

-Benjamin, W. (1995). Gesammelte Briefe. Vol. I. Frankfurt a. M.: Suhrkamp.

-De Lara, F. (2007). Heidegger y el cristianismo de San Pablo y San Agustín. Eidos (7), 28-46.

-Eiland, H. \& Jennings, M. W. (2014). Walter Benjamin. A Critical Life. Cambridge: Harvard University Press.

-Heidegger, M. (1978a). Frühe Schriften. Frankfurt a. M.: Klostermann.

-Heidegger, M. (1978b). Aus der Erfahrung des Denkens. Frankfurt a. M.: Klostermann.

-Heidegger, M. (1993). Grundprobleme der Phänomenologie (1919/1920). Frankfurt a. M.: Klostermann (hay trad. cast. en (2014), Problemas fundamen-tales de la fenomenología (1919/1920). Madrid: Alianza, 2014).

-Heidegger, M. (1995a). Pbänomenologie des religiösen Lebens. Frankfurt a. M.: Klostermann.

-Heidegger, M. (1995b). Ontologie. Hermeneutike der Faktizität. Frankfurt a. M.: Klostermann (hay trad. cast. en (1999), Ontología. Hermenéutica de la facticidad. Madrid: Alianza). 
-Heidegger, M. (1996). Anmerkungen zu Karl Jaspers Psychologie der Weltanschauungen (1919/1921). En Wegmarken. (págs. 3-44). Frankfurt a. M.: Klostermann (hay trad. cast. en (2000), Hitos. Madrid: Alianza).

-Heidegger, M. (1999). Zur Bestimmung der Philosophie. Frankfurt a. M.: Klostermann (hay trad. cast. parcial en (2005), La idea de la filosofía y el problema de la concepción del mundo. Barcelona: Herder).

-Heidegger, M. \& Jaspers, K. (1992). Briefwechsel 1920-1963. München/ Frankfurt: Piper/Klostermann.

-Ott, H. (1986). Der Habilitant Martin Heidegger und das von Schaezler'sche Stipendium. Ein Beitrag zur Wissenschaftsförderung der katholischen Kirche. Freiburger Diözesanarchiv, 106, 141-160.

-Ott, H. (1993): Las raíces católicas del pensamiento de Heidegger. En J. M. Navarro Cordón y R. Rodríguez (Eds.): Heidegger o el final de la filosofía. (págs. 163-174). Madrid: Editorial Complutense.

-Ott, H. (2004). Martin Heidegger und seine Beziehungen zur GörresGesellschaft zur Pflege der Wissenschaft im katholischen Deutschland. En A. Denker, H. Zaborowki y H.-H. Gander (Eds.), Heidegger und die Anfänge seines Denkens (págs. 197-200; Heidegger-Jahrbuch, vol. 1). Freiburg/München: Alber.

-Ott, H. (2005). Martin Heidegger und seine Beziehungen zur GörresGesellschaft. Historisches Jahrbuch. 125, 169-173.

-Regehly, T. (2002). Historische und erfüllte Zeit - Walter Benjamins Kritik an Heideggers Antrittsvorlesung über den "Zeitbegriff in der Geschichtswissenschaft” (1916)". En N. Lesniewski (Ed.), Die Zeit Heideggers (págs. 141152). Frankfurt/Berlin/Bern/Wien: Peter Lang.

-Thomä, D. (2003). Heidegger und der Nationalsozialismus. En D. Thomä (Ed.), Heidegger Handbuch (págs. 141-162). Stuttgart: Metzler.

-Trawny, P. (2014). Heidegger und der Mythos der jüdischen Weltverschwörung. Frankfurt a. M.: Klostermann.

-Vargas, M. (2012). El problema del tiempo histórico y la imagen dialéctica en Walter Benjamin. Revista latinoamericana de filosofía, 38 (1), 85-108.

-Wolfe, J. (2013). Heidegger's Schatology. Theological Horizons in Martin Heidegger's Early Work. Oxford: Oxford University Press.

Sumario: Introducción; 1. Identificación de los gestos políticos; 1.1. La conferencia de habilitación de 1915 y las primeras publicaciones de Heidegger; 1.2. Los primeros cursos friburgueses. "Ciencia y reforma de la universidad"; 1.3. "Caminos para el debate". El rol político de la filosofía en la ontohistoria; 2. Análisis crítico de los tres momentos; Conclusión; Referencias. 